\title{
On the Paper "The Effect of Proper Nonlinearity of the Medium on the Propagation of Ultimately Short Pulses in an Array of Carbon Nanotubes" by N.N. Yanyushkina and M.B. Belonenko
}

\author{
N. N. Rosanov \\ Vavilov State Optical Institute, St. Petersburg, 199034 Russia \\ St. Petersburg National Research University of Information Technologies, Mechanics, and Optics, \\ St. Petersburg, 197101 Russia \\ e-mail:nrosanov@yahoo.com \\ Received April 17, 2012
}

DOI: $10.1134 / \mathrm{S} 0030400 \mathrm{X} 13010220$

The subject of [1] is the evolution of ultimately short electromagnetic pulses in a nonlinear medium including a set of carbon nanotubes. Quantitative results were obtained by a numerical solution of onedimensional (the field depends on time $t$ and on a single-longitudinal-coordinate $x$ ) nonlinear wave equation (7) (the references are given to formulas from [1]). Here, the $x$ coordinate was chosen for the evolutional variable and wave equation (7) was complemented by boundary conditions corresponding to specifying field (the longitudinal component of the vector potential) $B(0, t)$ and the derivative of this function with respect to the coordinate $\partial B / \partial x(0, t)(8)$ at the boundary of the nonlinear medium (at $x=0)$.

In our opinion, such a formulation of the problem of describing the propagation of ultimately short pulses in a nonlinear medium is incorrect. Indeed, radiation with a wide spectrum in a nonlinear medium inevitably generates a counterpropagating wave; reflection from the boundary of the medium is present together with the volume reflection, which was also mentioned by the authors themselves in the third section. Therefore, the field at the boundary of the medium is composed of two fields, the first of which corresponds to the pulse incident to the boundary from outside and the second of which corresponds to the counterpropagating radiation caused by the reflection from the nonlinear medium. In a correct formulation, only the field of the incident pulse (the function $B(x, 0)$ and $\partial B / \partial t(x, 0)$, which is equivalent to specifying the electric and magnetic strengths of the electromagnetic field at initial time instant $t=0$ ) should be considered as specified. Here, the total field at the boundary of the medium at following time instants is a priori unknown and is to be determined. In the presence of counterpropagating waves, only time can serve as a correct evolutional variable. At the initial time instant, when the incident radiation pulse moves in a vacuum to the boundary of the medium but does not reach it, the function $B(x, 0)$ is specified; the derivative with respect to time for the pulse propagating in the vacuum in the positive direction of the $x$ axis is expressed via the derivative of this function with respect to the coordinate

$$
\partial B / \partial t(x, 0)=-c \partial B / \partial x(x, 0),
$$

where $c$ is the speed of light in a vacuum. This is the approach that was implemented, e.g., in [14] in the References of the considered paper. The choice of the coordinate as an evolutional variable would be possible in the absence of inverse volume reflection, which is truly negligible in the case of long quasi-monochromatic radiation pulses but not in the considered case of ultimately short pulses. This choice could be justified in an extremely artificial situation in which hypothetical devices detect the temporal profile of the reflected radiation at the boundary and then the boundary is supplied with an additional radiation that provides the required characteristics of the total field. However, this scheme is hardly of interest, at least as applied to ultimately short pulses.

\section{REFERENCES}

1. N. N. Yanyushkina and M. B. Belonenko, Opt. Spektrosk. 114 (1), 157 (2013).

Translated by A. Nikol'skii 